Sobre o closing lemma de classe $C^{r}$

Bernardo Paschoarelli Veiga Gomes 


\title{
Sobre o closing lemma de classe $C^{r}$
}

\author{
Bernardo Paschoarelli Veiga Gomes
}

Orientador: Prof. Dr. Carlos Teobaldo Gutierrez Vidalón

Dissertação apresentada ao Instituto de Ciências Matemáticas e de Computação da Universidade de São Paulo, como parte dos requisitos para obtenção do título de Mestre em Ciências na área: Matemática.

\section{USP - São Carlos}

Fevereiro/2006 
Aos meus pais com amor... 


\section{Agradecimentos}

Agradeço ao Prof. Dr. Carlos Gutierrez, pela orientação, direcionamento e contribuições para a realização deste trabalho,

Aos meus pais,

Aos funcionários da biblioteca, e demais funcionários do ICMC,

Aos colegas de curso e laboratório,

A FAPESP, pelo auxílio financeiro.

E a todos, que de alguma forma me ajudaram a concluir este trabalho. 


\section{Abstract}

In this work, we study some partial results related to the $C^{r}$-closing lemma, on bidimensional manifolds, for $1 \leq r \leq \infty$. For the torus $T^{2}$, we classify the $C^{r}$-vector fields with a non-trivial recurrent point $p$, in two types, one of which we call the constant type inspired by the terminology of continued fractions. This classification depends on the holonomy map defined by $X$ around $p$. If $X \in X^{r}\left(T^{2}\right)$ has finitely many singularities, and $X$ is not of constant type, we prove the existence of a vector field $Y$ arbitrarily close to $X$ (in the $C^{r}$ topology) having a periodic trajectory through $p$. For a bidimensional, compact and orientable manifold $M$ we show that the the $C^{r}$ vector fields on $M$ having a non-trivial wandering point $p$ fall in two types, one of which we call $\mathcal{B}$, and which also depends on the holonomy map around $p$ induced by $X$. If $X$ has finitely many singularities, and $X$ is of $\mathcal{B}$-type, we prove the existence of a vector field $Y$ arbitrarily close to $X$ (in the $C^{r}$-topology) having a periodic trajectory through $p$. 


\section{Resumo}

Neste trabalho, estudamos alguns resultados parciais relacionados ao closing lemma de classe $C^{r}$ em variedades bidimensionais para $1 \leq r \leq \infty$. Para o toro $T^{2}$, classificamos os campos vetoriais possuindo um ponto recorrente não-trivial $p$ em dois tipos, um dos quais chamaremos de tipo constante, inspirado na terminologia das frações contínuas. Esta classificação depende da transformação de holonomia induzida por $X$ ao redor de $p$. Se $X \in X^{r}\left(T^{2}\right)$ tem un número finitos de singularidades, e $X$ não é de tipo constante, demonstramos a existência de um campo vetorial $Y$, arbitrariamente próximo de $X$ (na topologia $C^{r}$ ), tendo uma trajetória periódica por $p$. Para uma variedade bidimensional, orientável e compacta $M$, mostramos que os campos vetoriais de classe $C^{r}$ sobre $M$ tendo um ponto errante não-trivial $p$ se dividem em dois tipos, um dos quais chamaremos de tipo $\mathcal{B}$, e que também depende da transformação de holonomia ao redor de $p$ induzida por $X$. Se $X$ tem um número finito de singularidades, e é de tipo $\mathcal{B}$, nós mostramos a existência de um campo vetorial $Y$, arbitrariamente próximo de $X$ (na topologia $\left.C^{r}\right)$, tendo uma trajetória periódica por $p$. 


\section{Sumário}

Introdução 1

1 Fluxos em superfícies $\quad 3$

1.1 Closing Lemma e algumas de suas consequências . . . . . . . . . . . . . . . . . 3

1.2 Transformações de primeiro retorno . . . . . . . . . . . . . . . . 4

2 Frações Contínuas, Rotações e IET's 9

2.1 Rotações . . . . . . . . . . . . . . . . . . . . . . . 9

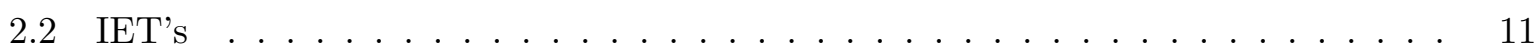

2.3 Condições de Minimalidade . . . . . . . . . . . . . . . . . . . . . . . 13

3 Closing lemma em variedades bidimensionais $\quad 15$

3.1 Closing lemma no toro $T^{2} \ldots \ldots \ldots \ldots \ldots \ldots$. . . . . . . . . . . . . . . . .

3.2 Closing lemma em variedades bidimensionais f . . . . . . . . . . . . . 24

A Noções básicas da Teoria dos Sistemas Dinâmicos $\quad 27$

B Noções básicas da Teoria Ergódica $\quad 29$

$\begin{array}{ll}\text { Bibliografia } & 31\end{array}$ 


\section{Introdução}

Esta dissertação visa estudar alguns resultados referentes ao closing lemma de classe $C^{r}$ em variedades bidimensionais, suaves, compactas e orientáveis, para $1 \leq r \leq \infty$. Segue o enunciado do closing lemma de classe $C^{r}$ :

Sejam $M$ uma variedade suave e compacta, $1 \leq r \leq \infty$ inteiro, $X \in X^{r}(M)$ (resp. $f \in$ Diff $f^{r}(M)$ ) e $p$ um ponto não-errante de $X$ (resp. $f$ ). Então existe um $Y \in X^{r}(M)$ (resp. $\left.g \in \operatorname{Diff}^{r}(M)\right)$ arbitrariamente próximo de $X,\left(\right.$ resp. $f$ ) na topologia $C^{r}$ tal que $p$ é ponto periódico de $Y$ (resp. de $g$ ).

Este trabalho é estruturado em três capítulos. No primeiro capítulo discutiremos o enunciado acima e algumas de suas motivações.

No segundo capítulo, apresentaremos alguns conceitos necessários para as demonstrações do terceiro capítulo, como a definição de fração contínua e a sua relação com os retornos próximos de uma rotação irracional. Definiremos também as transformações de intercâmbio de intervalos, analisaremos algumas de suas propriedades básicas e estudaremos a dinâmica do operador de Rauzy.

Por fim, no terceiro capítulo, demonstraremos os principais resultados desta dissertação. Descreveremos um argumento utilizado nos trabalhos de Gutierrez e Pugh, que permite reduzir a demonstração do closing lemma de classe $C^{r}$ à demonstração de uma proposição auxiliar. Feito isto, aplicaremos os conceitos desenvolvidos no capítulo 2, através de um resultado de Gutierrez, conhecido como teorema da estrutura. Este resultado possibilita classificar os fluxos em $T^{2}$ com pontos recorrentes não triviais em fluxos de tipo constante e de tipo não constante. Por meio desta classificação, é possível utilizar o teorema de estrutura para demonstrar o closing lemma de classe $C^{r}$ para uma classe grande de fluxos do toro, os denominados fluxos de tipo não-constante. Subsequentemente, utilizaremos uma versão mais geral do teorema de estrutura para estender os resultados obtidos para campos no toro $T^{2}$ à uma classe grande de fluxos em variedades bidimensionais, suaves e compactas, os chamados fluxos de tipo $\mathcal{B}_{k}$. Para estes campos, obtemos uma resposta afirmativa ao closing lemma de classe $C^{r}$. 


\section{Capítulo 1}

\section{Fluxos em superfícies}

\subsection{Closing Lemma e algumas de suas consequências}

O closing lemma foi levantado inicialmente no estudo realizado por Peixoto da classificação dos campos estruturalmente estáveis em superfícies compactas, e tem o seguinte enunciado:

Sejam $M$ uma variedade suave e compacta, $r \geq 1$ inteiro, $X \in X^{r}(M)$ (resp. $f \in \operatorname{Diff}^{r}(M)$ ) e $p$ um ponto não-errante de $X$ (resp. f). Então existe um $Y \in X^{r}(M)$ (resp. $g \in \operatorname{Diff}^{r}(M)$ ) arbitrariamente próximo de $X,\left(r e s p\right.$. f) na topologia $C^{r}$ tal que p é ponto periódico de $Y$ (resp. g).

campo $X$ sobre uma superfície orientável hiperbólicas, a seguinte alternativa: ou $p$ é periódico, ou $p$ une duas selas.

Consideraremos agora o caso $r=0$, cuja demonstração, mesmo para o caso $n$-dimensional é trivial. Como a trajetória de um ponto não-errante $p$ retorna arbitrariamente próxima de $p$, podemos conectar um destes pontos próximos de $p$ a $p$ por um arco quase paralelo ao campo. É fácil ver que por uma perturbação localizada, temos um novo campo $Y$ tal que o arco anterior faz parte de uma órbita periódica por $p$ (esta perturbação é definida dentro de uma caixa de fluxo, conceito a ser definido neste capítulo). No entanto, $Y$ em geral não está $C^{1}$-próximo de $X$.

Em [Pg2], Pugh formulou e demonstrou o closing lemma de classe $C^{1}$. Uma versão mais forte da afirmação acima, conhecida como closing lemma localizado, tem o seguinte enunciado:

(Closing Lemma localizado) Sejam $M$ uma variedade suave, compacta, $r \geq 1$ um inteiro, $X \in \chi^{r}(M)$ e p um ponto não-errante de $X$. Para qualquer vizinhança $V$ de $p$ em $M$ e qualquer vizinhança $\mathcal{V}$ de $X$ em $X^{r}(M)$, existe uma perturbação de $X$, com suporte em $V$, dada por um 
campo $Y \in \mathcal{V}$, de forma que $Y$ tenha uma órbita fechada por $V$.

Ainda em [Pg1], Pugh mostrou de fato a versão localizada do closing lemma de classe $C^{1}$.

Como estamos lidando com perturbações, é importante definir precisamente a topologia sobre $X^{r}(M)$, que é naturalmente identificado com o espaço $C^{r}\left(M, \mathbb{R}^{2}\right)$, já que estes dois espaços são homeomorfos. Muniremos $C^{r}\left(M, \mathbb{R}^{2}\right)$ com uma estrutura de um espaço de Banach, definindo uma norma \|\|$_{r}$.

Seja $\mathcal{V}=\left\{V_{1}, \ldots, V_{k}\right\}$ uma cobertura de $M$ por abertos, onde cada aberto está contido no dominio de uma carta local $\left(x_{i}, U_{i}\right)$ com $x_{i}\left(U_{i}\right)=B(2)$ e $x_{i}\left(V_{i}\right)=B(1)$, onde $B(1)$ e $B(2)$ são, respectivamente, as bolas de raios 1 e 2 e centradas na origem de $\mathbb{R}^{2}$.

Para $f \in C^{r}\left(M, \mathbb{R}^{2}\right)$, denotamos $f^{i}=f \circ x_{i}^{-1}: B(2) \rightarrow \mathbb{R}^{2}$. Definimos

$$
\|f\|_{r}=\max _{i} \sup _{u \in B(1)}\left\{\left\|f^{i}(u)\right\|,\left\|d f^{i}(u)\right\|, \ldots,\left\|d^{r} f^{i}(u)\right\|\right\} .
$$

Observação 1.1.1. Como demonstrado em [S-T], se p for um ponto não-errante e não-recorrente, então $\gamma_{p}$ conecta uma ou duas singularidades de $X$, como na figura 1.1. Além disto, existe um anel aberto $U$ e um ponto $q \in U$ cujo $\alpha$-limite ou $\omega$-limite contém $\gamma_{p}$. Neste caso é muito fácil produzir uma órbita periódica que intercepta qualquer vizinhança de p para perturbações arbitrariamente $C^{r}$-pequenas de $X$.

Exemplo 1.1.1. Defina um campo $X_{0}$ em $\mathbb{R}^{2}$ por $X(x, y)=(y,-x)$. As órbitas do fluxo gerado por $X_{0}$ são círculos centrados na origem, ou a própria origem. Agora defina o campo $X$ como o produto $f X_{0}$, onde $f \in C^{\infty}\left(\mathbb{R}^{2}, \mathbb{R}\right)$ vale zero em $P=(0,1), f$ assume valores positivos em $\mathbb{R}^{2}-\{P\}$ e $f$ tem norma $C^{\infty}$ menor do que um dado $\epsilon>0$. As órbitas de $X$ são as mesmas que as órbitas de $X_{0}$, a menos do círculo $C$ de raio 1 . De fato, este círculo contém duas órbitas de $X$; o ponto singular $P$ e $C-\{P\}$. É fácil ver que todo ponto q pertencente a $C-\{P\}$ é não-errante, e vale que $\omega(q)=P$. Portanto, q não é um ponto recorrente. O closing lemma de classe $C^{\infty}$ é válido neste caso: dado $\epsilon>0, f+\frac{\epsilon}{\|X\|}$ assume valores estritamente positivos em $C$, e portanto todo ponto de $\mathbb{R}^{2}$ está contido numa órbita periódica de $Y=\left(f+\frac{\epsilon}{2\|X\|}\right) X_{0}$, e além disto, $\|X-Y\|_{\infty}<\epsilon$.

\subsection{Transformações de primeiro retorno}

A teoria dos sistemas dinâmicos discretos está presente no estudo dos fluxos na análise das transformações de primeiro retorno. Estas são definidas numa vizinhança de uma variedade transversal a uma órbita periódica de um fluxo contínuo $X$. 
Ao longo deste texto, um segmento $C$ denotará a imagem de um mergulho suave de um intervalo fechado em uma variedade $M$. Segmentos de $M$, como arcos de trajetória de $X$, serão denotados como intervalos (fechados, semi-abertos ou abertos) na forma $[p, q)$.

Além disto, $M$ denotará uma variedade bidimensional, suave e compacta, e $\varphi: \mathbb{R} \times M \rightarrow M$ um fluxo contínuo sobre $M$. A semitrajetória positiva (resp., negativa) de p será denotada por $\gamma_{p}^{+}=\{\varphi(t, p)\}_{t \geq 0}$ (resp. $\left.\gamma_{p}^{-}=\{\varphi(t, p)\}_{t \leq 0}\right)$, e a trajetória $\gamma_{p}^{+} \cup \gamma_{p}^{-}$de $p$, por $\gamma_{p}$.

Se $p \in M e[a, b] \subset \mathbb{R}$, diremos que $\varphi([a, b] \times\{p\})$ é um arco de trajetória por $p$. O ponto $p \in M$ será dito um ponto regular de $\varphi$ caso não seja um ponto fixo de $\varphi$.

Definição 1.2.1 (Caixa de Fluxo). Seja $N$ uma subvariedade de $M$ disjunta do conjunto dos pontos fixos de $\phi . N \subset M$ é chamada de caixa de fluxo de $X$ caso existam um retângulo $[a, b] \times[c, d] \subset \mathbb{R}^{2}$ e um homeomorfismo $\theta:[a, b] \times[c, d] \rightarrow M$ tais que para todo $s \in[c, d]$, o conjunto $\theta([a, b] \times\{s\})$ seja um arco de trajetória de $\phi$ por $\theta(s)$. Neste caso também chamaremos $\theta$, ou $(N, \theta)$ de caixa de fluxo. Se $x=\theta(0,0)$, dizemos que $N$ é uma caixa de fluxo por $x$.

Observação Segue do teorema do fluxo tubular que se p for um ponto regular de $\phi$, sempre existe uma caixa de fluxo por $p$.

Definição 1.2.2. Sejam $C \subset M$ um um segmento (ou um círculo) de classe $C^{1}$ e $X \in X^{r}(M)$. Seja $I: C \rightarrow M$ a inclusão do segmento na variedade. Diremos que $C$ é transversal a $X$ caso para todo ponto $p \in C$, o conjunto $\left\{X(p), D_{p} I(p)\right\}$ seja uma base do espaço tangente de $M$ em $p$.

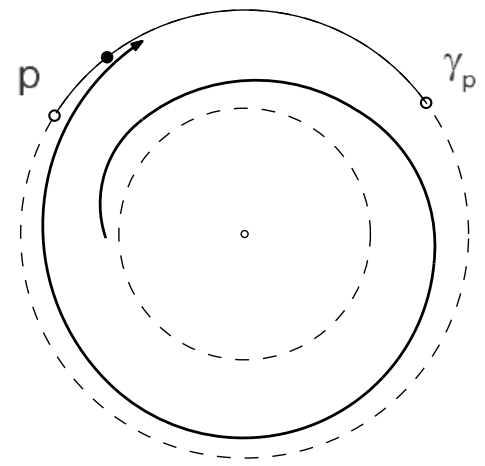

Figura 1.1:

O seguinte lema será utilizado no capítulo 3:

Lema 1.2.1. Seja $\Sigma$ um intervalo aberto contendo um ponto recorrente não-trivial $p$ de um fluxo contínuo $\varphi: \mathbb{R} \times M \rightarrow M$. Se $\Sigma$ for transversal a $\varphi$, então existe um círculo $C$ transversal a $\varphi$, tal que $C \cap \Sigma$ contém um intervalo aberto passando por $p$. 
No caso orientável, a demonstração é a seguinte: construimos duas caixas de fluxo, $\left(N_{1}, \theta_{1}\right.$ e $\left(N_{2}, \theta_{2}\right)$, a primeira contendo $\Sigma$, (utilizando o teorema do fluxo tubular longo), e a segunda contendo o arco de trajetória de $\phi$ que liga $p$ primeira interseç̧ão de $p$ com $\Sigma$, Tp. O círculo transversal é construindo unindo o subintervalo $[p, T p]$ de $\Sigma$ com o arco de trajetória que liga $p$ a Tp. O caso não-orientável é mais sutil, e sua demonstração está em [Pe2].

Definição 1.2.3. Seja $C$ uma curva transversal a $\varphi$. A transformação de retorno de Poincaré $T: \operatorname{Dom}(T) \rightarrow C$ é definida em $\operatorname{Dom}(T):=\{x \in C$ tal que existe $T>0$ satisfazendo $\varphi(T, x) \in$ $C\}$.

Observação 1.2.1. Segue do teorema do fluxo tubular que Dom $(T)\}$ é um subconjunto aberto de $C$, e $T$ tem a mesma classe de diferenciabilidade que $\varphi$. Definindo uma nova transformação de retorno na imagem de uma componente conexa de $\operatorname{Dom}(T)$, vemos que $\operatorname{Dom}\left(T^{i}\right)$ é aberto para todo $i \in \mathbb{Z}-\{0\}$.

\section{Resultados Parciais}

A importância do closing lemma de classe $C^{r}$ é justificada pelas suas diversas aplicações à Teoria Genérica, e à Teoria da Bifurção. Alguns teoremas importantes, como o teorema da densidade geral de classe $C^{1}$ de Pugh [Pg2], que afirma que em $X^{1}(M)$ é genérico que $\overline{\operatorname{Per}(X)}=$ \{pontos errantes não-triviais\}, também estão associado ao closing lemma.

O closing lemma de classe $C^{1}$ é utilizado para demonstrar o fato dos campos de MorseSmale serem densos em $X^{1}(M)$, sendo $M$ uma variedade bidimensional, orientável ou não. Uma resposta afirmativa ao closing lemma de classe $C^{r}$ para $r>1$ estenderia este resultado para o caso $r>1$.

Outro resultado relevante é o closing lemma ergódico de classe $C^{1}$ de Mañé, que é utilizado para caracterizar os difeomorfismos estruturalmente estáveis em variedades bidimensionais [Man].

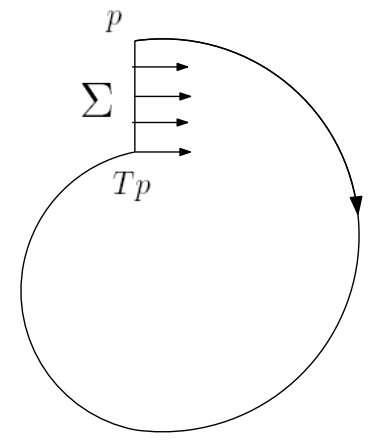

Figura 1.2: 
Para campos em variedades bidimensionais $M$ como o disco, onde vale o teorema da curva de Jordan, ou mais precisamente, o teorema de Poincaré-Bendixon, o closing lemma é trivialmente satisfeito, pois não existem pontos recorrentes não-triviais. Suponha que exista um ponto $\omega$ recorrente $p \in M . p$ é regular, logo existe uma seção transversal ao fluxo $\Sigma$ que contém $p$. Seja $T p$ o primeiro ponto em que o arco da trajetória positiva de $p$ intersecta $\Sigma$, e I o subintervalo de $\Sigma$ determinado por estes dois pontos. Então o arco de trajetória que liga $p$ a Tp unido com I é um círculo topológico, (ver figura 1.2), e pelo teorema da curva de Jordan, $M$ se divide em duas componentes conexas, $A$ e B. Suponha que B contém $\omega(p)$. Como existe uma caixa de fluxo $N$ em $p$, depois de um tempo $T>0$, todos os pontos $p$ de $N \cap B$ saem de $N$, portanto $\{\phi(t, p)\}_{t>T}$ está a uma distância positiva de p, mais especificamente, a uma distância maior ou igual que a distância entre os compactos $B-N$ e $\{p\}$. Isto impede que $p$ seja um ponto recorrente, já que $d(p, \omega(p))>0$. Desta forma, chegamos a um absurdo.

Outro resultado nesta direção é um teorema de Marckley [Mar], que mostra que a garrafa de Klein também não apresenta pontos de recorrência não-trivial. 


\section{Capítulo 2}

\section{Frações Contínuas, Rotações e IET's}

\subsection{Rotações}

O estudo da recorrência é de importância central na teoria de sistemas dinâmicos. Nesta seção estudaremos a recorrência para as rotações irracionais do círculo; isto é; quão frenqüentemente e quão próximo uma órbita retorna a uma vizinhança de seu ponto inicial. A análise das rotações no círculo tem suas raízes na teoria dos números e envolve tópicos como frações contínuas e condições diofantinas. Além disto, como será visto no próximo capítulo, a transformação de primeiro retorno em um segmento transversal a um campo $X$ no toro é semiconjugada a uma rotação $R_{\theta}$. Veremos então que se $\theta$ satisfizer uma propriedade da teoria dos números, o closing lemma de classe é valido para o campo $X$.

Se $x \in \mathbb{R} / \mathbb{Z}$, escrevemos $\|x\|=\inf _{k \in \mathbb{Z}}|x-k|$.

Definiremos para uma rotação irracional $R_{\alpha}$ em $\mathbb{R} / \mathbb{Z}$ uma sequência de retorno próximos $\left\{q_{i}\right\}_{i} \in \mathbb{N}$ como sendo uma sequência de inteiros onde para cada $i \in \mathbb{N}$ valha que $\left\|R^{q_{i}}(0)\right\| \leq$ $\left\|R^{j}(0)\right\|$, para $0<j<q_{i}$. Esta seqûencia existe pelo fato de $R_{\alpha}$ ser minimal.

Seja $\xi \in \mathbb{R}$. Defina $[x]$ como a parte inteira de um real $x$ e frac $(x)$ como sua parte fracionária, de forma que $x=[x]+\operatorname{frac}(x)$. Defina também, para $x$ real e não-nulo, $G(x)=\frac{1}{x}-\left[\frac{1}{x}\right]$.

Seja agora $\alpha \in(0,1)$ irracional. Então para todo $n$ natural, $G^{n}($ frac $(\alpha)) \neq 0$. Portanto podemos definir a sequência $a_{0}=[\alpha]$ e $a_{n}=\left[G^{n-1}(\operatorname{frac}(\alpha))^{-1}\right]$.

Simbolicamente, escrevemos

$$
\alpha=a_{0}+\frac{1}{a_{1}+\frac{1}{a_{2}+\frac{1}{a_{3}+\ldots}}} .
$$

Para simplificar a notação, escrevemos também $\alpha=\left[a_{0}, a_{1}, a_{2}, a_{3}, \ldots\right]$. Dizemos então que $a_{n}$ é o enésimo quociente parcial de $\alpha$, e que a fração reduzida

$$
\frac{p_{n}}{q_{n}}=\left[a_{0}, \ldots, a_{n}\right]=a_{0}+1 /\left(a_{1}+\left(1 /\left(a_{2}+\ldots+1 /\left(a_{n-1}+1 / a_{n}\right) \ldots\right)\right)\right.
$$


é o enésimo convergente principal de $\alpha$.

Teorema 2.1.1. (Teorema das Frações Contínuas) Se $\left\{p_{n} / q_{n}\right\}$ denota a sequência de convergentes principais de $\alpha$, então $\lim _{n \rightarrow \infty} \frac{p_{n}}{q_{n}}=\alpha$.

Sejam $\left\{a_{n}\right\}$ a sequência de quocientes parciais de $\alpha, \widehat{q_{n} \alpha}=q_{n} \alpha-p_{n}$, e para $\theta \in \mathbb{R}$, seja $I_{n}+\theta$ o subintervalo compacto de $\mathbb{R}$ com pontos extremos $\theta$ e $\theta+q_{n} \alpha$. Utilizaremos a notação simplificada $I_{n}+0=I_{n}$.

A proposição abaixo descreve a sequência de retornos próximos da rotação $R_{\alpha}$ :

Proposição 2.1.1. Se $|q|>0$ é um inteiro tal que $|q|<q_{n+1}$, então $\|q \alpha\|<\left\|q_{n} \alpha\right\|$. De forma recíproca, se $n \geq 1, q_{n+1}$ é o menor inteiro com módulo maior que $\left|q_{n}\right|$ tal que $\left\|q_{n} \alpha\right\|<\left\|q_{n+1} \alpha\right\|$.

Proposição 2.1.2. As sequências $\left\{p_{n}\right\}$ e $\left\{q_{n}\right\}$ satisfazem as seguintes relações: (i) $p_{n}=$ $a_{n} p_{n-1}+p_{n-1}$ para $n \geq 2, p_{0}=a_{0}, p_{1}=a_{0} a_{1}+1$. (ii) $q_{n}=a_{n} q_{n-1}+p_{n-2}$ para $n \geq 2$, $q_{0}=1, q_{1}=a_{1}$.

Proposição 2.1.3. Se $n \geq 1, \mid q_{n} \alpha\left\|=a_{n}\right\| q_{n-1} \alpha\|+\| q_{n} \alpha \|$, e se $n \geq 3$ :

$$
a_{n}=\left[\frac{\left\|q_{n-2}\right\|}{\left\|q_{n-1}\right\|}\right]
$$

Proposição 2.1.4. Seja $\theta \in \mathbb{R}$. Para todo $n \geq 2$, temos que $q_{n+1}<2 q_{n}$. Além disto:

(a) Os intervalos módulo $1\left\{R_{j \alpha}\left(I_{n}+\theta\right)\right\}_{0 \leq j \leq q_{n}}$, onde $j$ é um inteiro, são dois a dois disjuntos, exceto pelo fato de que

$$
\left(I_{n}+\theta\right) \cap\left\{R_{q_{n} \alpha}\left(I_{n}+\theta\right)\right\}=\left\{\widehat{q_{n} \alpha}+\theta\right\} ;
$$

(b) Os números reais $\theta, \widehat{q_{n} \alpha}+\theta$ e $\widehat{2 q_{n} \alpha}$ estão ordenados na reta real da seguinte maneira:

$$
\theta<(-1)^{n} \widehat{q_{n} \alpha}+\theta<(-1)^{n} \widehat{2 q_{n} \alpha}+\theta<(-1)^{n} \widehat{q_{n-2} \alpha}+\theta
$$

A demonstração do seguinte lema está em [Si]:

Lema 2.1.1. Para todo $n \in \mathbb{N}, n \geq 2$, os elementos de $\left\{A_{i, n} \| i \in\left\{0,1,2, \ldots,\left[a_{n} / 2\right]\right\}\right\}$ são conjuntos dois a dois disjuntos, onde

$$
A_{i, n}=\bigcup_{j=2 i q_{n}}^{(2 i+1) q_{n}} R_{j \alpha}\left(I_{n}\right)=\bigcup_{j=0}^{q_{n}} R_{j \alpha+2 i \widehat{q_{n} \alpha}}\left(I_{n}\right)
$$

Proposição 2.1.5. Seja $\left\{i_{k}\right\}$ uma sequência estritamente crescente de números naturais ímpares. Suponha que $\lim _{k \rightarrow \infty} a_{i_{k}}=\infty$. Dado um subconjunto finito $\left\{x_{1}, x_{2}, \ldots, x_{m}\right\}$ de $\mathbb{R} / \mathbb{Z}$, existe um $n_{0} \in \mathbb{N}$ tal que se $i_{k} \geq n_{0}$, então para algum $\theta \in\left(\theta,\left\|q_{i_{k}} \alpha\right\|\right), \bigcup_{j=0}^{q_{n}} R_{j \alpha+\theta}\left(I_{i_{k}}\right)$ é disjunto de $\left\{x_{1}, x_{2}, \ldots, x_{m}\right\}$. 
Demonstração Tomamos $n_{0} \in \mathbb{N}$ tal que, para todo $i_{k} \geq n_{0}, a_{i_{k}+1}>2(m+1)$. Segue do lema anterior que os elementos de $\left\{A_{j, i_{k}} \mid j=1,2, \ldots, m+1\right\}$ são conjuntos dois a dois disjuntos. Logo, para algum $\sigma \in\{1,2, \ldots, m+1\}, A_{j, i_{k}}-\bigcup_{j=0}^{q_{i_{k}}} R_{j \alpha+\theta}\left(I_{i_{k}}\right)$ é disjunto de $\left\{x_{1}, x_{2}, \ldots, x_{m}\right\}$, e portanto, satisfaz as condições da proposição.

Definição 2.1.1. Sejam $\alpha \in(0,1)$, e $\left[a_{1}, a_{2}, \ldots\right]$ sua expansão em frações contínuas. Dizemos que $\alpha$ é de tipo constante caso exista uma constante $C>0$ tal que $a_{i}<C$ para todo $i$ natural. Caso contrário, dizemos que $\alpha$ é de tipo não-constante.

Proposição 2.1.6. $O$ conjunto $C:=\{x \in(0,1) \mid x$ é de tipo constante $\}$ tem medida de Lebesgue nula.

\section{$2.2 \quad$ IET's}

As tranformações de intercâmbio de intervalos, termo aqui abreviado por IET's; do inglês interval exchange transformations, são as transformações mais simples que preservam medida em um intervalo semi-aberto $[0,1)$. Além disto, as IET's generalizam as rotações do círculo. A inclusão deste tópico neste texto se justifica pela utilidade das IET's no estudo de transformações de primeiro retorno definidas por fluxos em superfícies compactas.

A seguir definimos uma tranformação de intercâmbio de intervalo.

Sejam $I=[0,1), m>1$ e $\alpha=\left(\alpha_{1}, \ldots, \alpha_{m}\right) \in \mathbb{R}^{m}$, satisfazendo $\alpha_{i}>0$ para todo $i \in\{1, \ldots, m\}$ e $\sum_{i=1}^{m} \alpha_{i}=1$. $\alpha$ é denominado vetor de probabilidade. Adotaremos a notação $\Delta_{m}$ para representar o conjunto de todos os vetores de probabilidade de $\mathbb{R}^{m}$.

Fixando $\alpha \in \Delta_{m}$, definimos os números $\beta_{0}=0, \beta_{i}=\sum_{j=1}^{i} \alpha_{j}$ e os conjuntos $I_{i}=\left[\beta_{i-1}, \beta_{i}\right)$, onde $i \in\{1, \ldots, m\}$. Para cada $\tau \in S(m)$, definimos $\alpha^{\tau}=\left(\alpha_{\tau^{-1}(1)}, \ldots, \alpha_{\tau^{-1}(m)}\right)$. É fácil ver que $\alpha^{\tau}$ é outro vetor de probabilidade. Sejam $\beta_{0}^{\tau}=0, \beta_{i}^{\tau}=\sum_{j=1}^{i} \alpha_{i}^{\tau}$, e $I_{i}^{\tau}=\left[\beta_{i-1}^{\tau}, \beta_{i}\right)$. Desta forma, $\alpha$ e $\tau$ induzem as partições $\left\{I_{i}\right\}_{i=1}^{m}$ e $\left\{I_{i}^{\tau}\right\}_{i=1}^{m}$ de $[0,1)$.

Definição 2.2.1. Sejam $\alpha \in \Delta_{m}$ e $\tau \in S(m)$ permutação. Defina $T:[0,1) \rightarrow[0,1)$ por

$$
T x=x-\beta_{i-1}+\beta_{\tau(i)-1}^{\tau}
$$

$T$ é chamada de uma $(\alpha, \tau)$-IET sobre $m$ intervalos.

Observação 2.2.1. É fácil ver que uma rotação é uma IET que age sobre dois intervalos. Isto segue do fato de que se $\theta \in \mathbb{R}$, ao identificarmos o círculo com o intervalo $[0,1), R_{\theta}:[0,1) \hookleftarrow$ coincide com a IET $T_{\alpha, \pi}$ definida por $\alpha=(\theta, 1-\theta) \in \mathbb{R}^{2}$ e $\pi=(12)$. 
Observação 2.2.2. Podemos definir uma IET sobre qualquer intervalo semi-aberto $[p, q)$, com $p \leq q$ números reais, da seguinte forma: Na definiçôes acima tome $\alpha \in \mathbb{R}^{m}$ tal que $\alpha_{i}>0$, $\forall i \in 1, \ldots, m$ e $\sum_{i=1}^{m} \alpha_{i}=p-q$.

A seguir, $T$ denotará uma IET arbitrária, e sem perda de generalidade, consideraremos seu domínio como $[p, q)=[0,1)$.

Observação 2.2.3. Note que os pontos de descontinuidade de $T$ pertencem ao conjunto $\left\{\beta_{1}, \ldots, \beta_{m}\right\}$ e $T$ é contínua em $\beta_{i}$ se e somente se $\tau(i+1)=\tau(i)+1$. Ou seja; $T$ é descontínua em $\beta_{i}$ se e somente se $\left(T \beta_{i-1}, T \beta_{i}\right)$ não surgem nesta ordem no conjunto ordenado $\left\{\beta_{0}^{\tau}, \ldots, \beta_{m-1}^{\tau}\right\}$. Dizemos que $T$ está na forma padrão caso as descontinuidades de $T$ sejam somente os $\beta_{i}$ 's, ou de forma equivalente, se $\tau(i+1) \neq \tau(i)+1$ para todo $i \in 1, \ldots, m-1$. É fácil ver que qualquer IET pode ser escrita na sua forma padrão como uma IET sobre $n$ intervalos, com $n \leq m$.

Agora exibiremos um exemplo de uma IET sobre 3 intervalos:

Exemplo 2.2.1. Tomando $m=3, \alpha=(0.3 ; 0.6,0.1)$ e $\tau=(1,2,3) \mapsto(3,2,1)$, temos:

$$
\left\{\begin{aligned}
I_{1} & =[0,0.3) \\
I_{2} & =[0.3,0.9) \\
I_{3} & =[0,9.1) \\
I_{1}^{\tau} & =[0.7,1) \\
I_{2}^{\tau} & =[0.1,0.7) \\
I_{3}^{\tau} & =[0,0.1)
\end{aligned}\right.
$$

Por fim,

$$
T(x)=\left\{\begin{array}{cc}
x+0.7, & x \in[0,0.3) \\
x-0.2, & x \in[0.3,0.9) \\
x-0.9, & x \in[0.9,1)
\end{array}\right.
$$

O gráfico de $T$ está representado abaixo na figura 2.1.

1. $T$ restrita a $I_{i}$ é uma isometria sobre $I_{\tau(i)-1}^{\tau}$.

2. $T$ é invertível.

3. $T^{k}$ é uma IET para todo $k$ inteiro. 


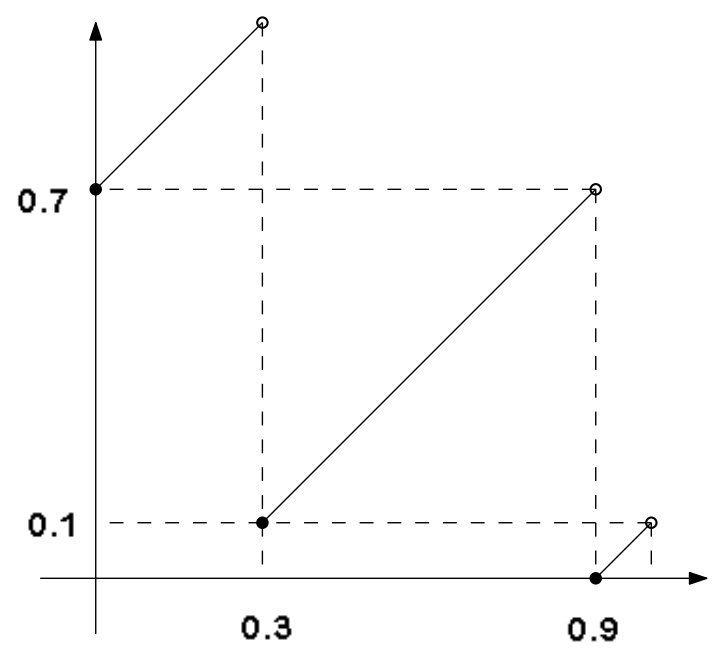

Figura 2.1:

\subsection{Condições de Minimalidade}

O resultado a seguir caracteriza as IET's minimais:

Teorema 2.3.1. (Oseledets-Keane)Seja T uma $(\alpha, \tau)$-IET. Então $T$ é minimal se e somente se $T$ satisfaz as chamadas condições de minimalidade (M1) e (M2):

M1 Se F é a união finita de intervalos semi-abertos cujos extremos estão contido no seguinte conjunto: $D^{\infty}=\bigcup_{n=0}^{m-1} \mathcal{O}(x) \cup\{1\}$ Então $T(F)=F$ implica $T(F)=F$ ou $T(F)=\emptyset$.

M2 $T$ não possui órbitas periódicas.

Considere os seguintes espaços de medida: $\Delta_{m}$ munido da medida medida de Lebesgue $(m-$ 1)-dimensional normalizada $d \mu$ e $S(m)$ munido da medida da contagem normalizada $d \delta$. Ao associarmos um elemento $(\alpha, \tau)$ de $\Delta_{m} \times S(m)$ a uma $(\alpha, \tau)$-IET, identificamos naturalmente o espaço de probabilidade $\left.\left(\Delta_{m} \times S(m)\right), d \mu \times d \delta\right)$ com o conjunto de todas as IET's sobre $n$ intervalos.

Definição 2.3.1. Sejam $E:[a, b) \rightarrow[a, b)$ uma IET e $c \in a, b)$. Definia a IET induzida em $[a, c), F$ da seguinte forma: $\operatorname{Dom}(F)=\left\{x \in[a, c): T^{N}(x) \in[a, c)\right.$ para algum $\left.N>0\right\}$.

Definição 2.3.2. Seja $E:[a, b) \rightarrow[a, b)$ uma IET. Dizemos que $[s, t) \subset[a, b)$ é uma aresta virtual orthogonal de $E$ se $E$ restrita $a[s, t)$ for contínua e $s \geq E(s) \geq E^{2}(s)=t$. Defina $\mathcal{B}_{k}$ como o conjunto das IET's $E:[a, b) \rightarrow[a, b)$ que possuam associadas a si uma sequência de pontos $b_{n} \rightarrow a$, tal que cada IET induzida $E_{n}:\left[a, b_{n}\right) \rightarrow\left[a, b_{n}\right)$ possui ao menos $\chi+k+3$ arestas virtuais ortogonais, duas a duas disjuntas. Denotaremos o conjunto $\bigcap_{k \geq 1} \mathcal{B}_{k}$ por $\mathcal{B}$. 
Lema 2.3.1. Para todo $m \geq 2, \Delta_{m} \times S(m)-\mathcal{B}$ é um conjunto de medida zero.

\section{Demonstração}

Como foi demonstrado por Veech [Vee] e Masur [Mas], o operador de Rauzy $\mathcal{R}: \mathcal{M} \rightarrow$ $\mathcal{M}$, definido em um conjunto de medida total $\mathcal{M}$ de $\Delta_{m} \times S(m)$ é ergódico e tem a seguinte propriedade $(\mathrm{P})$ :

(P) Dado $E \in \mathcal{M}$, existe uma sequência $\left\{\left[0, a_{n}\right)\right\}$ de sub-intervalos de $[0,1)$, tais que $a_{n} \rightarrow 0$ e se $\tilde{E}_{n}:\left[0, a_{n}\right) \rightarrow\left[0, a_{n}\right)$ denotar a IET induzida por $E$, então a menos de um reescalonamento, ${ }_{G} R^{n}(E)$ coincide com $E$. De forma mais precisa, $\mathcal{R}^{n}(z)=\left(1 / a_{n}\right) \tilde{E}\left(a_{n} z\right)$, para todo $z \in[0,1)$.

Dado $k \geq 0$, seja $A_{k}$ o conjunto dado por $E \in \Delta_{m} \times S(m)$ tais que para algum $a \in\left(16^{-k}\right.$ $\left.32^{-k}, 16^{-k}+32^{-k}\right), E(x)=a+x$, para todo $x \in\left[0, \frac{1}{2}\right]$. Observe que $A_{k}$ é aberto, e portanto tem medida positiva. Seja $\tilde{B}_{k}$ o conjunto dado por $E \in \mathcal{M}$ tal que a $\mathcal{R}$-órbita positiva de $E$ visita $A_{k}$ infinitas vezes. $\tilde{B}_{k}$ é $\mathcal{R}$ invariante, $\mathcal{R}$ é ergódico e $A_{k}$ tem medida positiva, portanto o complemento de $\tilde{B}_{k}$ tem medida zero. Logo o complemento de $\mathcal{B}=\bigcap_{k \geq 2} \tilde{\mathcal{B}}_{k}$ tem medida zero.

Observe que se uma IET $E \in A_{k}$, então $E$ tem mais do que $k$, duas a duas disjuntas, arestas virtuais ortogonais. Portanto, como $\mathcal{R}$ satisfaz $(P)$ acima, e como a $\mathcal{R}$-órbita positiva de qualquer $E \in \tilde{\mathcal{B}}$ visita $A_{k}$ um número infinito de vezes, obtemos que $\tilde{\mathcal{B}} \subset \mathcal{B}$. 


\section{Capítulo 3}

\section{Closing lemma em variedades bidimensionais}

A partir dos conceitos desenvolvidos nos capítulos anteriores, demonstraremos, baseado nos artigos [Gu3] e [Gu6] de Gutierrez, alguns resultados parciais acerca do closing lemma de classe $C^{r}$ para uma variedade bidimensional, suave, compacta e orientáveis, que ao longo deste texto, denotaremos por $M$. A primeira seção consiste da demonstração de um teorema acerca do closing lemma de classe $C^{r}$ para $M=T^{2}$, o toro bidimensional. A segunda seção por sua vez, generaliza os resultados da primeira seção para $M$ qualquer.

Antes, porém, se faz necessário demonstrar dois lemas.

Lema 3.0.2 (Lema da homotopia). Suponha que existe uma sequência $\left\{F_{n}\right\}_{n \in \mathbb{N}} \subset \operatorname{Diff} f^{r}(M)$ tal que $F_{n}\left(p_{n}\right)=p, \forall n \in \mathbb{N}$. Suponha também que $F_{n}$ tende para o difeomorfismo identidade na topologia $C^{r}$. Então, cada campo $\left(F_{n}\right)^{*}\left(X+t_{n} X^{\perp}\right)$ é de classe $C^{r}$ e possui uma órbita fechada por p. Além disto, $\left(F_{n}\right)^{*}\left(X+t_{n} X^{\perp}\right) \rightarrow X$ na topologia $C^{r}$.

Definição 3.0.3. Uma $\Sigma$-caixa de fluxo para $X$ é um subconjunto compacto $F \subset M$ cujo interior é uma caixa de fluxo para $X$, e cuja fronteira é um grafo, homeomorfo a figura do "oito", que é a união dos arcos de trajetória $[\bar{a}, \bar{c}]_{X}$ e $[\bar{a}, \bar{c}]$, (conectando $\bar{a}$ a $\bar{c}$ ), de $X$ e $X^{\perp}$, respectivamente. Nos referiremos a $[\bar{a}, \bar{c}]_{X}$ e $[\bar{a}, \bar{c}]$ como a aresta ortogonal e tangente, respectivamente, de $F$.

A figura 2.1 ilustra uma $\Sigma$-caixa de fluxo:

Definição 3.0.4. Sejam $X \in X^{r}(M), 1 \leq r \leq \infty$, e $p$ um ponto recorrente não-trivial de $X$. Dizemos que $X$ é $\Sigma$-fechado em $p$, caso exista um intervalo $\Sigma=[p, q)$ tangente a $X^{\perp}$, tal que para qualquer vizinhança $V$ de $p$, existe uma $\Sigma$-caixa de fluxo para $X$ tendo a sua aresta ortogonal contida em $\Sigma \cap V$. 


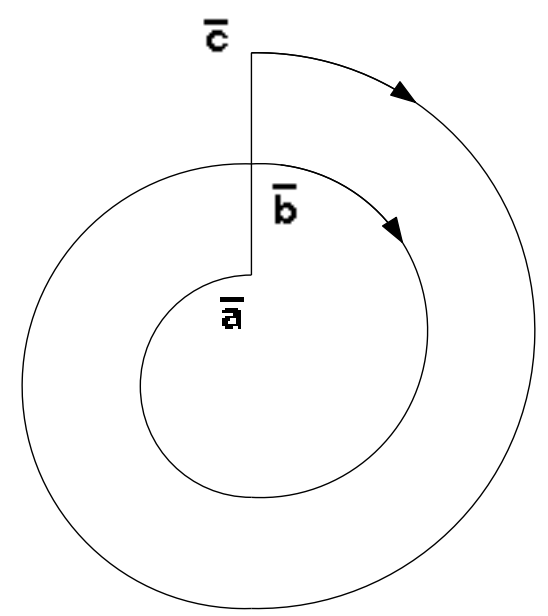

Figura 3.1:

Lema 3.0.3. Sejam $X \in X^{r}(M), 1 \leq \infty$, e $p \in M$ um ponto recorrente não trivial de $X$. Suponha que $X$ é $\Sigma$-fechado em $p$. Então existem sequências $t_{n} \rightarrow 0$, de números reais, $e$ $p_{n} \rightarrow p$, de pontos de $M$, tais que $X+t_{n} X^{\perp}$ possui uma trajetória fechada por $p_{n}$.

\section{Demonstração}

Como $X$ é $\Sigma$-fechado em $p$, existe um intervalo semi-aberto $\Sigma=[p, q)$ tangente a $X^{\perp}$,tal que dadas vizinhanças $\mathcal{V}$ de $X$, e $V$ de $p$, podemos escolher uma $\Sigma$-caixa de fluxo de $X, F \subset M$, e $\sigma>0$, tais que se $[\bar{c}, \bar{a}]_{X}$ e $[\bar{a}, \bar{c}]$ são as arestas tangente e ortogonal de $\partial F$, respectivamente, e $\bar{b}$ é o vértice de $\partial F$. Então:

(b1) $[\bar{a}, \bar{c}] \subset V$ e o fluxo $X$ entra em $F$ através do subintervalo fechado $[\bar{b}, \bar{c}]$ de $\Sigma$. Além disto, para todo $t \in[\sigma, \sigma], X(t):=X+t X^{\perp} \in \mathcal{V}$.

(b2) $X(-\sigma)$ e $X(-\sigma)$ possuem um arco de trajetória contido em $F$, ambos sendo seções transversais globais de $\left.X\right|_{F}$.

Continuaremos considerando o caso em que o fluxo de $X^{\perp}$ vai de $\bar{a}$ para $\bar{c}$. Seja $\Gamma$ o conjunto de todos os números reais $s \in[0, \sigma]$ tais que para $t \in[0, s]$ existe um arco de trajetória $[\bar{b}, \bar{a}(t)]_{X(t)}$ de $X(t)$, unindo $\bar{b}$ com $\bar{a}(t) \in[\bar{a}, \bar{b}]$. Neste caso, note que o referido arco de trajetória de $\overline{c a}$ por $X+X^{\perp}$ não escapa da $\Sigma$-caixa de fluxo, posto que se isto acontecesse, teríamos que para algum $\mu$ e $p \in \partial F, X(p)$ e $\left(X+^{+}\right)(p)$ seriam tangentes, o que é um absurdo. Segue que $\bar{a}(0)=\bar{a}$, e que $\bar{a}(t)$ depende continuamente de $t$. Como visto na figura 3.2 abaixo, quando $t \in \Gamma$, estas condições determinam $\bar{a}(t)$ e também que $[\bar{b}, \bar{a}(t)]_{X(t)}$ seja transversal a $X$. Além disto, por (b2), $\Gamma=\left[0, \sigma_{1}\right]$ é um intervalo fechado, $\bar{a}\left(\sigma_{1}\right)=\bar{b}$, e portanto $X+\sigma_{1} X^{\perp}$ apresenta uma órbita fechada por $\bar{b}$.

Das observações acima segue que: 


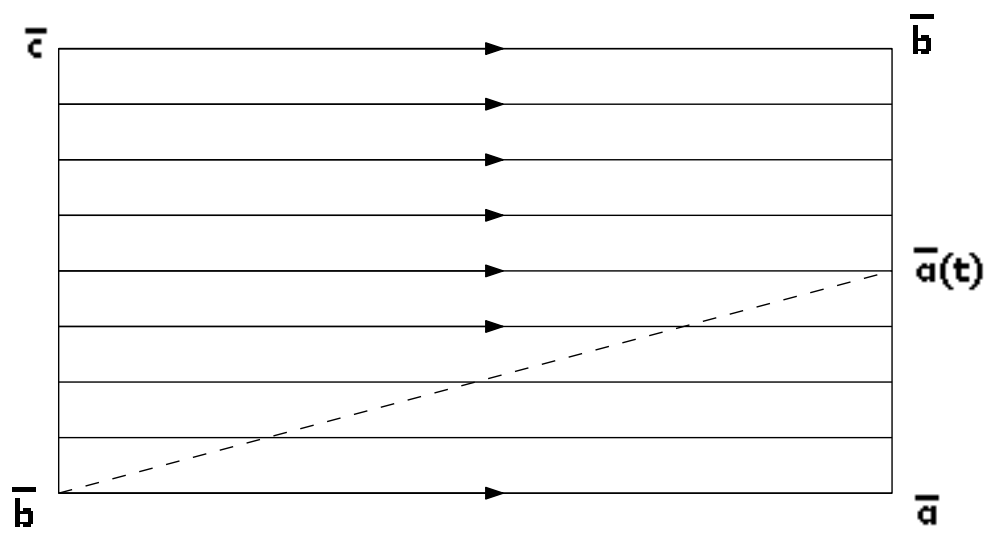

Figura 3.2:

Teorema 3.0.2. Sejam $X \in X^{r}(M)$ e $1 \leq r \leq \infty$. Seja $p$ um ponto recorrente não-trivial de $X$. Suponha que $X$ é $\Sigma$-fechado em $p$. Então existe $Y \in X^{r}(M)$ arbitrariamente próximo de $X$ e tendo uma trajetória fechada por p.

Supondo que o fluxo sobre $M$ possui um número finito de singularidades, digamos $K \in \mathbb{N}$, a existência de $\Sigma$-caixas arbitrariamante próximas de $p$ garantem que podemos tomar $(K+1)$ $\Sigma$-caixas em qualquer vizinhança, desta forma garantindo que ao menos uma $\Sigma$-caixa contenha uma singularidade. De fato, este argumento será utilizado adiante.

\subsection{Closing lemma no toro $T^{2}$}

Nesta seção mostraremos que o closing lemma de classe $C^{r}$ é válido no toro $T^{2}$ para os fluxos que são de tipo não-constante. Mais adiante definiremos o que é um fluxo de tipo não-constante. O objetivo desta seção é demonstrar o seguinte teorema:

Teorema 3.1.1 (Teorema A). Sejam $X \in X^{r}\left(T^{2}\right),(r \geq 2)$, contendo $K \in \mathbb{N}$ singularidades. Se $X$ for de tipo não-constante e $p$ for um ponto recorrente não-trivial de $T^{2}$, então existe um campo vetorial $Y$, de classe $C^{r}$, arbitrariamente próximo de $X$ na topologia $C^{r}$, tal que $Y$ possui uma trajetória fechada por $p$.

Como veremos, o teorema acima é conseqüência da combinação dos lemas 3.1.1, 3.1.3 e do teorema abaixo, devido a Gutierrez [Gu2], que não demonstraremos aqui, e que está relacionado à suavização de fluxos contínuos em variedades bidimensionais:

Teorema 3.1.2 (Teorema de Estrutura). Seja $\phi: \mathbb{R} \times T^{2} \rightarrow T^{2}$ um fluxo contínuo sobre o toro $T^{2}$ que possui ao menos uma trajetória recorrente não trivial. Então existe um compacto 
$\varphi$-invariante $\Omega \subset T^{2}$ tal que qualquer trajetória recorrente não-trivial de $\varphi$ é densa em $\Omega$. Além disto, o conjunto $\mathcal{C}(\Omega)=\left\{\right.$ Círculos orientados de $T^{2}$ que interceptam $\Omega$ e são transversais a $\left.\varphi\right\}$, é não-vazio e satisfaz as seguintes propriedades:

(a) Considere $T: C \rightarrow C$ como sendo a transformação de primeiro retorno em $C$ com relação a $\varphi$, e o conjunto $\mathbb{R} / \mathbb{Z}$ munido com a orientação positiva usual. Então dado $C \in \mathcal{C}(\Omega)$, existem uma rotação geométrica não-periódica $R(C): \mathbb{R} / \mathbb{Z} \rightarrow \mathbb{R} / \mathbb{Z}$ e uma função contínua, monótona, preservando orientação e de grau um, $h: C \rightarrow \mathbb{R} / \mathbb{Z}$, que semiconjuga $T$ com $R(C)$. Ou seja; para todo $x \in \operatorname{Dom}(T), h \circ T(x)=R(C) \circ h(x)$. Além disto, se $h_{1}, h_{2}: C \rightarrow \mathbb{R} / \mathbb{Z}$ são transformações que possuem as mesmas propriedades de $h$, então para alguma rotação geométrica $R_{\alpha}: \mathbb{R} / \mathbb{Z} \rightarrow \mathbb{R} / \mathbb{Z}, h_{1}=R_{\alpha} \circ h_{2}$.

(b) Se o fluxo $\phi$ tem $n$ pontos fixos e $T$ e $h$ são como em (a), então existe $\left\{x_{1}, x_{2}, \ldots, x_{m}\right\} \subset$ $\mathbb{R} / \mathbb{Z}$, com $m \leq n$, tal que

$$
h^{-1}\left(\mathbb{R} / \mathbb{Z}-\left\{x_{1}, x_{2}, \ldots, x_{m}\right\}\right) \subset \operatorname{Dom}(T)
$$

Antes de prosseguir, demonstraremos dois lemas auxiliares e incluiremos uma definição:

Lema 3.1.1. Sejam $X$ e $Y$ espaços métricos, $X$ compacto, $A \subset X$ aberto e $f: X \rightarrow Y$ função contínua. Então o conjunto $W=\left\{y \in Y j f^{-1}(y)\right.$ está contido em $\left.A\right\}$ é um subconjunto aberto de $Y$.

\section{Demonstração}

Mostraremos que $Y-W$ é fechado, ou seja; dada uma seqüência $\left\{y_{n}\right\} \subset Y-W$ convergindo para algum $y \in Y$, temos que $y \in Y-W$. Pela definição de $W$ existe uma sequência de pontos $\left\{x_{n}\right\}$ em $X-A$ tal que $f\left(x_{n}\right)=y_{n}$ para todo $n$. Como $X$ é compacto e $A$ é aberto, $X-A$ também é compacto, o que implica a existência de uma subseqüência $\left\{x_{n_{k}}\right\}$ em $X-A$ tendendo para algum $x \in X-A$. Pela continuidade de $f, \lim _{k} y_{n_{k}}=f(x)$. Por sua vez, $\lim _{n} y_{n}=y$, o que implica que $f(x)=y$. Já que $x \notin A$, temos que $y \notin W$, como queríamos.

Lema 3.1.2. Seja $C$ uma curva suave e $\mathbb{R} / \mathbb{Z}$ munido da orientação induzida pelo intervalo $[0,1]$. Sendo $f: C \rightarrow Y$ função contínua e monótona, a imagem inversa de um ponto é um subconjunto conexo de $C$.

\section{Demonstração}

Suponha por absurdo que existe $x \in \mathbb{R} / \mathbb{Z}$ tal que $h^{-1}(\{x\})$ seja a união de dois fechados disjuntos de $C$, digamos $J$ e $K$, de forma que, sem perda de generalidade, que $\inf J<\inf K$. A igualdade na expressão anterior não vale pois $J$ e $K$ são conjuntos fechados. Então existe um ponto $P \in C$, entre $J$ e $K$. Agora, se $Q$ pertence a $J$, e $R$ pertence a $K$, segue do fato de $h$ 
preservar ordem que $h(Q) \leq h(P)$ e $h(P) \leq h(R)$. Então $x \leq h(P) \leq x \Rightarrow h(P)=x$. Absurdo, pois $P$ foi escolhido de forma que $P \notin h^{-1}(x)$.

O teorema de estrutura tem como implicação o teorema a seguir:

Corolário 3.1.1. Suponha as condições e notação do teorema de estrutura. Dado $C \in \mathcal{C}(\Omega)$, o subconjunto de $C$ formado pelos pontos recorrentes por ambos lados é denso em $C \cap \Omega$.

\section{Demonstração}

Sejam $h: C \rightarrow \mathbb{R} / \mathbb{Z}, T: \operatorname{Dom}(T) \rightarrow C$ e $R(C): \mathbb{R} / \mathbb{Z} \rightarrow \mathbb{R} / \mathbb{Z}$ como no item (a) do teorema de estrutura.

Se $x \in C \cap \Omega$, pela transversalidade de $C$ existe uma caixa de fluxo $\theta$ por $x$ tal que $\theta(\{0\} \times$ $[-1,1])=\Omega \cap C$. Como existe um ponto recorrente $q \in M$ com órbita densa em $\Omega \cap C$, existe um ponto $x \in \gamma_{q}$ no interior da caixa de fluxo $\theta$ pertencente a um arco de trajetória de $q$. Por definição, $\theta$ é homeomorfismo, portanto, $x=\theta(\tau, \xi)$, para algum $(\tau, \xi) \in[-1,1] \times[-1,1]$. Pela definição de caixa de fluxo, $p^{\prime}:=\theta(0, \xi)$ pertence a $\gamma_{q} \cap C$. Sem perda de generalidade, suporemos que $p^{\prime}$ é $\omega$-recorrente. Pelo lema A.0.1, $p^{\prime}$ também é um ponto recorrente não-trivial.

Defina $A_{0}=\mathbb{R} / \mathbb{Z}$ e $A_{i}=\left\{x \in \mathbb{R} / \mathbb{Z} \mid h^{-1}(x)\right.$ está contido em $\left.\operatorname{Dom}\left(T^{i}\right)\right\}$, para $i \in \mathbb{Z}-\{0\}$. Para um $i \in \mathbb{Z}$ qualquer, $\operatorname{Dom}\left(T^{i}\right)$ é um aberto de $C$, fato que combinado com o lema 3.1.1 implica que $A_{i}$ é um subconjunto aberto de $\mathbb{R} / \mathbb{Z}$.

Agora, fixe $i \in \mathbb{Z}$. Então $p:=T^{i}\left(p^{\prime}\right)$ e toda a sua órbita positiva pertencem a $\operatorname{Dom}\left(T^{i}\right)$.

O próximo passo consiste em demonstrar que $A_{i}$ é denso em $\mathbb{R} / \mathbb{Z}$. De fato, dado $x_{0} \in \mathbb{R} / \mathbb{Z}$, existe uma subsequência $\left\{x_{n}\right\}_{n \geq 1}$ da $R(C)$-órbita positiva de $h(p)$ tal que $\lim _{n} x_{n}=x_{0}$. Defina agora $p_{n}$ como sendo um ponto de $\gamma \cap C$ que satisfaz $h\left(p_{n}\right)=h(q)=x_{n}$. Suponha que $q$ seja outro ponto que satisfaz estas mesmas propriedades. Então $q \in \gamma_{p_{n}} \Rightarrow q=T^{k}\left(p_{n}\right)$, para algum $k \in \mathbb{Z}$. Logo $h\left(T^{k}\left(p_{n}\right)\right)=h\left(p_{n}\right) \Rightarrow R^{k}(C) \circ h\left(p_{n}\right)=h\left(p_{n}\right)$. Como $R(C)$ é aperiódica, $k=0$, o que implica $q=p_{n}$.

Denote por $J_{n}$ a componente conexa de $p_{n}$ em $\operatorname{Dom}\left(T^{i}\right) \cdot p_{n}$ é ponto recorrente não-trivial, logo pelo argumento acima, $h$ é injetora sobre $J_{n} \cap \gamma_{p}$. Portanto $h\left(J_{n}\right)$ é um conjunto conexo e infinito, ou seja; um intervalo de $\mathbb{R} / \mathbb{Z}$. Desta forma, os intervalos que são levados em pontos por $h$ contêm somente pontos errantes.

Pelo lema 3.1.2, $h^{-1}(x)$ é um ponto, ou senão, um intervalo de $\mathcal{C}$. Como imagens inversas de pontos distintos são conjuntos disjuntos, e além disto, uma curva pode conter no máximo uma quantidade enumerável de intervalos abertos, temos que existe no máximo uma quantidade enumerável de pontos em $\mathbb{R} / \mathbb{Z}$ cuja imagem inversa é um intervalo. Desta forma, o conjunto $\mathbb{R} / \mathbb{Z}-\mathcal{U}$, é enumerável, onde $\mathcal{U}=\left\{x \in \mathbb{R} / \mathbb{Z} j h^{-1}(x)\right.$ é um conjunto unitário $\}$. Agora, tome um ponto $y_{n} \in \mathcal{U} \cap h\left(J_{n}\right) \cap B\left(p_{n}, 1 / n\right)$. Desta forma, $h\left(q_{n}\right)$ é unitário e $h^{-1}\left(q_{n}\right) \in J_{n} \subset \operatorname{Dom}\left(T_{i}\right)$, 
isto é, $q_{n} \in A_{i}$. Além disto, $\lim _{n} y_{n}=\lim _{n} x_{n}=x_{0}$, o que mostra que $A_{i}$ é denso. Defina $\beta=\mathcal{U} \cap\left(\bigcap_{i \in \mathcal{Z}} A_{i}\right)$. Pelo teorema da categoria de Baire, $\beta$ também é denso em $\mathbb{R} / \mathbb{Z}$.

Seja $I \cap \gamma \cap C$ um aberto de $\gamma \cap C$, onde $I$ é um intervalo de $C$. Mostraremos que existe um ponto recorrente por ambos lados em $I \cap \gamma \cap C$. Por argumentos anteriores, $h(I)$ contém um intervalo aberto $J$, e portanto um elemento $y \in \beta \cap I$. A $R(C)$-órbita positiva de $y$ intercepta cada componente conexa de $J-\{x\}$ uma quantidade infinita de vezes. Como $h$ preserva ordem, a $T$-órbita positiva de $h^{-1}(y)$ também intercepta cada componente conexa de $J-\{x\}$ uma quantidade infinita de vezes.

O mesmo argumento pode ser repetido para a $R(C)$-órbita negativa de $y$. Portanto, $h^{-1}(y)$ é um ponto recorrente por ambos lados.

Observação 3.1.1. Não demonstraremos este fato, cuja demonstração está em [Gu4], mas uma das conseqüências do teorema de estrutura é o seguinte: Se $\varphi$ possui uma órbita recorrente nãotrivial $\gamma$ e se $C \in C(\Omega)$, então $\gamma$ é densa em $C$, ou $\overline{C \cap \gamma}$ é um conjunto de Cantor.

Definição 3.1.1. Seja $M$ uma variedade munida com a orientação usual e uma métrica suave Riemanniana $\langle$,$\rangle . Dados X \in X^{r}(M), 1 \leq r \leq \infty$, definimos $X^{\perp} \in X^{r}(M)$ como o campo que satisfaz as seguintes propriedades:

$\mathbf{a}\langle X, X\rangle=\left\langle X^{\perp}, X^{\perp}\right\rangle, e$

b Sempre que $p \in M$ for um ponto regular de $X$, o par ordenado $\left(X(p), X^{\perp}(p)\right)$ é uma base ortogonal positiva de $T_{p}(M)$ (fixada uma orientação de $M$ ).

Sejam $\phi: \mathbb{R} \times T^{2} \rightarrow^{2}$ um fluxo contínuo sobre $T^{2}$ e $\Sigma$ um segmento aberto, orientado $e$ transversal a $\phi$. Diremos que $\Sigma$ é $\varphi$-positivo, (respectivamente, $\phi$-negativo), se para tempo crescente, o fluxo $\phi$ atravessa $\Sigma$ como na figura abaixo. Seja $C$ um círculo transversal a $\phi$. Diremos que $C$ é $\phi$-positivo, (respectivamente, $\phi$-negativo), caso cada subintervalo de $C$, com a orientação

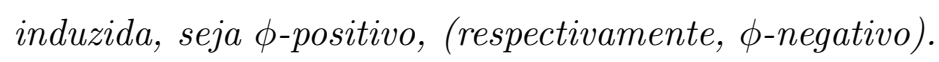

Teorema 3.1.3. Suponha as condições do teorema de estrutura (3.2). Dados dois círculos $C$ e $\tilde{C}$ em $\mathcal{C}(\Omega)$, existem $m, n \in \mathbb{N}$ tais que para todo $i \in \mathbb{N}, a_{i+n}=\tilde{a}_{i+m}$, onde $\left\{a_{k}\right\}$ e $\left\{\tilde{a}_{k}\right\}$ denotam as seqüências dos quocientes parcias dos números de rotação $\alpha$ e $\tilde{\alpha}$, de $R(C)$ e $R(\tilde{C})$, respectivamente. Além disto, $m-n$ é um inteiro par se e somente se $C$ e $\tilde{C}$ são simultaneamente $\phi$-positivos ou $\phi$-negativos.

\section{Demonstração}


Introduziremos a seguinte notação: Se $z \in T^{2}$ e $w=X_{t}(z)$, para algum $t \in(0, \infty), \Gamma(z, w)$ denotará o arco de trajetória de $X$ começa em $z$ e termina em $w$.Quando $p \in M$ e $q \in \varphi(\tau, p)$, para algum $\tau>0$, o arco de trajetória conectando $p$ com $q$ será denotado $\Gamma(p, q)$.

Pelo corolário 3.1.1, existe um ponto recorrente por ambos lados $p \in \Omega \cap C$. Denote por $T: C \rightarrow C$, (respectivamente, por $\widetilde{T}: \widetilde{C} \rightarrow \widetilde{C}$ ), a transformação de Poincaré induzida por $\varphi$, e por $\Sigma_{n}$, (respectivamente, por $\widetilde{\Sigma}_{n}$ ), com $n \in \mathbb{N}-\{0\}$, o subintervalo aberto de $C$, (respectivamente), de $\widetilde{C}$ ), que possui como pontos extremos $T^{-q_{n}}(p)$ e $T^{q_{n}}(p)$, e que contém $p$. Sejam $\left\{q_{n}\right\}$, (resp. $\left\{\widetilde{q}_{n}\right\}$ ), a sequência dos denominadores dos convergentes pricipais de $\alpha$, (resp. $\widetilde{\alpha}$ ). Pelo teorema de estrutura, podemos assumir que $h(p)=0$, apenas alterando a semiconjugação por meio de uma composição com a rotação $R_{-h(p)}$. Da proposição 2.1.1, segue que:

(1) $\left\|R_{\alpha}^{q_{n+1}}(h(p))-h(p)\right\|<\left\|R_{\alpha}^{j}(h(p))-h(p)\right\|$, para $0<j<q_{n+1}$. Isto implica que $R_{\alpha}^{j}(h(p)) \notin$ $\left[-R_{\alpha}^{q_{n}}(h(p)), R_{\alpha}^{q_{n}}(h(p))\right]$, para $0<j<q_{n+1}$. Pelo fato de $h$ preservar ordem, segue que; $T^{j}(p) \notin \Sigma_{q_{n}}$, para $0<j<q_{n+1}{ }^{1}$. Como $\Gamma\left(p, T^{q_{n+1}}(p)\right) \cap \Sigma_{q_{n}} \subset\left\{p, T(p), \ldots, T^{q_{n}}(p), T^{q_{n}+1}(p)\right\}$, $\Gamma\left(p, T^{q_{n+1}}\right) \cap \Sigma_{q_{n}}=\left\{p, T^{q_{n+1}}(p)\right\}$. De forma recíproca, temos que se $m \in \mathbb{N}$ for grande o suficiente e $\Gamma\left(p, T^{m}(p)\right) \cap \Sigma_{q_{m}}=\{p\}$, então $m$ é igual a algum $q_{m}$.

Observe também que:

(2) Se $C$ e $\tilde{C}$ são homotópicas, e a homotopia preserva as orientações de $\Sigma$ e $\tilde{\Sigma}$, então $R(C)=R(\tilde{C})$.

Sejam $q$ o primeiro ponto de $\gamma_{p}^{+}$que intersecta $\tilde{C}$, e $B$ a caixa de fluxo cujo interior contém $\Gamma(p, q)$. Podemos utilizar uma homotopia com suporte em $B$ para transformar $\tilde{C}$ em um novo círculo, ainda denotado por $\tilde{C}$, mas agora satisfazendo:

(3) $C \cap \tilde{C}$ contém um intervalo $\Sigma$ que contém $p$. Sem perda de generalidade, por (1), podemos supor que vale (2). Seja $m$ tal que $\Sigma_{m} \subset C \cap \tilde{C}$. Por (1), podemos concluir que $p T^{m}(p) \cap \Sigma_{\tilde{q}_{m}}=$ $\{p\}$, então $m$ é igual a algum $q_{n}$. Logo $q_{n}=q_{m}$. Desta forma, como $\Gamma\left(p, T^{q_{n+1}}(p)\right) \cap \Sigma_{q_{n}}=$ $\left\{p, T_{q_{n+1}}(p)\right\}$ e $\Gamma\left(p, T^{\tilde{q}_{n+1}}(p)\right) \cap \Sigma_{\tilde{q_{n}}}=\left\{p, T^{q_{n+1}}(p)\right\}$, temos que $T^{q_{n+1}}(p)=T^{q_{m+1}}(p) \Rightarrow n+1=$ $m+1$. De forma indutiva, $n+i=m+i$ para todo $i \in \mathbb{N}$.

Para $j, l \in \mathbb{N}$, defina $\mathcal{U}_{j}\left(\Sigma_{l}\right)$ e $\tilde{\mathcal{U}}_{j}\left(\tilde{\Sigma}_{l}\right)$ como as cardinalidades dos conjuntos $\Gamma\left(p, T^{q_{j}}(p)\right) \cap \Sigma_{l}$, e $\Gamma\left(p, T^{\tilde{q}_{j}}(p)\right) \cap \Sigma_{l}$, respectivamente. Por 2.1.3, e pelo fato de $R(C)$ e $R(\tilde{C})$ serem unicamente ergódicas, podemos aplicar o teorema ergódico de Birkhoff para concluir que para os naturais $n$ e $m$ acima, e para todo $i \in \mathbb{N}$, vale que:

$$
a_{n+1}=\left[\frac{2 q_{n+i-2} \alpha}{2 q_{n+i-1} \alpha}\right]=\left[\frac{h\left(\Sigma_{q_{n+i-2}}\right)}{h\left(\Sigma_{q_{n+i-1}}\right)}\right]=\left[\lim \frac{\mathcal{U}_{q_{n+i+j}}\left(\Sigma_{q_{n+i-2}}\right)}{\mathcal{U}_{q_{n+i+j}}\left(\Sigma_{q_{n+i-1}}\right)}\right]=
$$

\footnotetext{
${ }^{1}$ Note que $-R_{\alpha}^{j}(h(p))=R_{\alpha}^{-j(h(p))}$
} 


$$
=\left[\lim _{j} \frac{\tilde{\mathcal{U}}_{\tilde{q}_{m+i+j}}\left(\tilde{\Sigma}_{\tilde{q}_{m+i-2}}\right)}{\tilde{\mathcal{U}}_{\tilde{q}_{m+i+j}}\left(\tilde{\Sigma}_{\tilde{q}_{m+i-1}}\right)}\right]=\left[\frac{2 \tilde{q}_{n+i-2} \alpha}{2 \tilde{q}_{n+i-1} \alpha}\right]=\tilde{a}_{m+i} .
$$

Utilizando (3) e o item (b) de 2.1.4, podemos concluir que se $q_{n}-q_{m}$ for par, então $T^{q_{n}}(p)$ e $T^{q_{m}}(p)$ estão na mesma compontente conexa de $C-\{p\}$. Logo $m-n$ é par se, e somente se as orientações de $\Sigma$, induzidas pelas orientações de $C$ e $\tilde{C}$ são as mesmas.

É imediato pela parte final do teorema acima que

Corolário 3.1.2. Sejam $\varphi$ e $\mathcal{C}(\Omega)$ como no teorema de estrutura. A propriedade de $R(C)$ ser de tipo constante, (respectivamente, não-constante), onde $C \in \mathcal{C}(\Omega)$, não depende de $C$ em particular, mas sim de $\varphi$.

O corolário acima justifica a definição a seguir:

Definição 3.1.2. Sejam $\varphi$ e $\mathcal{C}(\Omega)$ como no teorema de estrutura. Diremos que $\phi$ é de tipo constante (respectivamente, não constante), caso exista algum $C \in(\Omega)$ tal que $R(C)$ seja de tipo constante (respectivamente, não constante).

Iniciamos agora a demonstração do teorema A.

(1) Suporemos que o ponto não-errante em questão é não-trivial: pois caso sua órbita fosse periódica, poderíamos tomar $X=Y$ no closing lemma. Como visto anteriormente, basta demonstrar o caso em que $p$ é recorrente não trivial. Assim, supondo as condições do teorema A, podemos tomar o ponto $p$ como recorrente não-trivial e $X$ como sendo de tipo não-constante. Fixando uma orientação para $T^{2}$, tome um intervalo suave, $X$-positivo e aberto $\Sigma$, passando por p. Ver figura 5.2.

Utilizando o lema 1.2.1 e o fato de que $X$ não é de tipo constante, podemos supor que a orientação de $T^{2}$ foi escolhida de forma que:

(2) Se $h$ e $R(C)$ são as transformações referidas no item (a) do teorema de estrutura e $\alpha$ é o número de rotação de $R(C)$, então existe um subsequência $\left\{a_{i_{k}}\right\}$ de $\left\{a_{i}\right\}$, a sequência de quocientes parciais de $\alpha$, tal que todo sufixo $i_{k}$ é um número ímpar. Também vale que $\lim _{k} a_{i_{k}}=\infty$. Seja $\mathcal{V}$ uma vizinhança de $X$ em $X^{r}\left(T^{2}\right)$. Suponha que $\Sigma$ tenha sido tomado tangente ${ }^{2}$ a $\gamma_{p}$ em $p$, e de forma que, para algum $\epsilon>0$ :

(3) Existe uma função contínua estritamente crescente $\sigma:[0, \epsilon) \rightarrow C$, satisfazendo $\sigma(0)=p$, e tal que para todo $\mu \in[0, \epsilon), X+\mu X^{\perp} \in \mathcal{V}$. Além disto, os segmentos $\sigma((0, \mu))$ e $\sigma((\mu, \epsilon))$, com a orientação induzida por $C$ são respectivamente $X+\mu X^{\perp}$-positivos, e $X+\mu X^{\perp}$-negativos, como na figura 3.1 :

\footnotetext{
${ }^{2}$ Esta construção é feita por meio de uma caixa de fluxo como a do exemplo da observação ??.
} 
Como $p$ é um ponto recorente por ambos lados, $h(\sigma[0, \epsilon))$ contém um intervalo $I$. Pelo corolário 3.1.1, podemos tomar um ponto recorrente por ambos lados $q \in h^{-1}(I) \cap \sigma(0, \epsilon)$. Novamente, podemos supor $h(q)=0$ e que existe um subconjunto finito $\left\{x_{1}, \ldots, x_{m}\right\} \subset \mathbb{R} / \mathbb{Z}$ tal que

(4) $\left\{x_{1}, \ldots, x_{m}\right\} \subset \operatorname{Dom}(T)$.

Sejam $\theta \in \mathbb{R}, r>0$ tais que o intervalo $[\theta, \theta+r]$ esteja propriamente contido em $h[0, \epsilon)$. Tome $n \in \mathbb{N}$ ímpar tal que $\widehat{q_{n-1} \alpha}<r$. Portanto Segue de (2) e das proposições 2.1.4 e 2.1.5, que $R_{\theta}\left(\left[0, \widehat{q_{n} \alpha}\right]\right)$ e $R_{\theta}\left(\left[\widehat{q_{n} \alpha}, \widehat{2 q_{n} \alpha}\right]\right)$ estão contidos em $h(\sigma[0, \epsilon))$.

(5) Além disto, $\bigcup_{j=0}^{q_{n}} R_{j \alpha+\theta}\left(\left[0, \widehat{q_{n} \alpha}\right]\right)$ é disjunto de $\left\{x_{1}, x_{2}, \ldots, x_{m}\right\}$.

Sob estas condições, o item (b) da proposição 2.1 .4 implica que se $p_{1} \in h^{-1}(\theta), p_{2} \in$ $h^{-1}\left(\widehat{q_{n} \alpha+\theta}\right)$ e $p_{3} \in h^{-1}\left(\widehat{2 q_{n} \alpha}+\theta\right)$. Como $n$ é ímpar:

$\widehat{2 q_{n} \alpha}+\theta>\widehat{q_{n} \alpha}+\theta>\theta$,

De forma que

$\sigma^{-1}\left(p_{3}\right)<\sigma^{-1}\left(p_{2}\right)<\sigma^{-1}\left(p_{1}\right)$.

Por (4) e (5), podemos supor que:

(6) $T^{q_{n}}\left(p_{1}\right)=p_{2}, T^{q_{n}}\left(p_{2}\right)=p_{3}$, e que $T^{q_{n}}\left(\overline{p_{1} p_{2}}\right)=\overline{p_{2} p_{3}}$.

Se $x, y \in \sigma([0, \epsilon)), \overline{x y}$, denotará o subintervalo de $\sigma([0, \epsilon))$ com a orientação induzida.

O conjunto $B=\bigcup_{x \in \overline{p_{1} p_{2}}} \Gamma\left(x, T^{q_{n}} x\right)$ pode ser pensado como uma caixa de fluxo com os seus dois intervalos colados em $p$. Em outras palavras, $B$ é uma $\Sigma$-caixa de fluxo.

Mostraremos agora que existe um $\mu_{0} \in(0, \epsilon)$ tal que $X+\mu_{0} X^{\perp}$ exibe uma órbita fechada por $p_{2}$. Dado $\mu \in\left(0, \sigma^{-1}\left(p_{2}\right)\right)$, denote por $A_{\mu} \subset \operatorname{int}(B)$ o arco de trajetória aberto e orientado de $X+\mu_{0} X^{\perp}$ que começa em $p$, e termina no ponto $x(\mu)$ na fronteira de $B$. As seguintes propriedades são satisfeitas: (7.1) Para todo $\mu \in\left(0, \sigma^{-1}\left(p_{2}\right)\right), \overline{p_{2} p_{1}}$ é um segmento $\left(X+\mu X^{\perp}\right)$ positivo e $\Gamma\left(p_{2}, p_{3}\right)$ e $\Gamma\left(p_{1}, p_{2}\right)$ são segmentos $\left(X+\mu X^{\perp}\right)$-negativos.

(7.2) Para todo $\mu \in\left[0, \sigma^{-1}\left(p_{3}\right)\right], \overline{p_{3} p_{2}}$ é um segmento $\left(X+\mu X^{\perp}\right)$-positivo.

(7.3) Para todo $\mu \in\left(\sigma^{-1}\left(p_{3}\right), \sigma^{-2}\left(p_{2}\right)\right), \overline{p_{3} \sigma(\mu)}$ é um segmento $\left(X+\mu X^{\perp}\right)$-negativo e $\overline{\sigma(\mu) p_{2}}$ é um segmento $\left(X+\mu X^{\perp}\right)$-positivo.

Das propriedades acima, segue que $A_{\mu}$ está bem dfinido, e que $x(\mu)$ é uma função contínua, monótona e estritamente crescente, de $\mu \in\left(0, \sigma^{-1}\left(p_{2}\right)\right)$ e que para algum $\mu_{0} \in\left(0, \sigma^{-1}\left(p_{2}\right)\right)$, $x\left(\mu_{0}\right)=p_{2}$. Desta forma, o teorema A está demonstrado. 


\subsection{Closing lemma em variedades bidimensionais}

Ao longo desta seção $M$ denotará uma variedade suave, orientável, compacta, bidimensional e $\chi$, sua característica de Euler.

O objetivo desta seção é demonstrar um teorema análogo ao Teorema A, tratando, porém, do caso mais geral em que $M$ é qualquer variedade bidimensional, compacta e orientável. Veremos que o comportamento assintótico de um ponto recorrente não-trivial $p$, de um campo vetorial $X \in$ $X^{r}(M)$, depende do comportamento assintótico da trajetória $\gamma_{p}$. Este itinerário é representado por uma IET, digamos $E=E(p)=E((p),[p, q))$, que é semiconjugada a uma transformação de primeiro retorno $T$ definida em um intervalo semi-aberto $[p, q)$ transversal ao fluxo. Na seção anterior, as IET's consideradas são aquelas que agem irredutivelmente sobre apenas dois intervalos: as rotações. Enunciaremos o fato de que o fato de $E(p,[p, q))$ pertencer ao conjunto $\mathcal{B}$ definido no capítulo 2 não depende do segmento transversal escolhido, mas sim do fluxo. Quando $E$ pertencer a $\mathcal{B}$, então será possível encontrar $\Sigma$-caixas de fluxo arbitrariamente próximas de $p$, onde campos arbitrariamente próximos de $X$ possuem uma órbita fechada.

Teorema 3.2.1 (Teorema B). Para algum conjunto $\mathcal{B}$ de medida total no conjunto das IET's vale a seguinte afirmação: Seja $X$ um campo vetorial de classe $C^{r}, 1 \leq r \leq \infty$, com um número finito de singularidades sobre uma superfície compacta orientável $M$. Dado um ponto recorrente não-trivial $p \in M$ de $X$, a transformação de primeiro retorno ao redor de $p$ é semi-conjugada a uma IET $E:[0,1) \rightarrow[0,1)$. Se $E \in \mathcal{B}$, então existe um campo vetorial $Y$, de classe $C^{r}$, arbitrariamente próximo de $X$ na topologia $C^{r}$, tal que $Y$ possui uma trajetória fechada por $p$.

Notas

(a) $\mathrm{O}$ conjunto $\mathcal{B}$ está definido na seção 2.3 .

(b)O teorema acima garante a validade do closing lemma de classe $C^{r}$ para uma classe de fluxos, mas não nos dá informações sobre o "tamanho" ou densidade desta classe de fluxos em $X^{r}(M)$. Novamente, apresentaremos um teorema de estrutura devido Gutierrez, que introduzirá as IET's citadas no capítulo 2, no contexto do estudo de fluxos:

Teorema 3.2.2 (Teorema de Estrutura). Seja $X \in X^{r}(M)$. Existe uma quantidade finita de trajetórias $\gamma_{p_{1}}, \gamma_{p_{2}} \ldots, \gamma_{p_{l}}$ de $X$ tais que se $\gamma_{p}$ é qualquer trajetória recorrente não-trivial, então $\overline{\gamma_{p}}={\overline{\gamma_{i}}}_{\text {}}$, para algum $i=1,2, \ldots, l$. Suponha que $X$ tem exatamente $K \in \mathbb{N}$ singularidades (o caso $K=0$ é permitido). Seja $p$ um ponto recorrente não-trivial de $X$. Tome um intervalo semiaberto $[p, q) \subset M$ transversal a $X$, tal que $p$ é um ponto de acumulação de $\gamma_{p} \cap(p, q)$. Denote por $P_{X}:[p, q) \rightarrow[p, q)$ a transformação de primeiro retorno induzida por $X$. Se $[p, q)$ for pequeno o suficiente, ele pode ser associado com $(p,[p, q))$, uma IET $E=E_{(p,[p, q))}:[0,1) \rightarrow[0,1)$, e uma 
transformação contínua, monótona e sobrejetiva $h:[p, q) \rightarrow[0,1)$ tal que $h(p)=0, h$ restrita a uma dada órbita de $P_{X}$ é injetiva, e para todo $x \in \operatorname{Dom}\left(P_{X}\right), E \circ h(x)=h \circ P(x)$. Além disto;

(a1) Existe um subconjunto $S \subset[0,1)$ de no máximo $\chi+K+2$ elementos tal que se $A$ for uma componente conexa de $[0,1)-S$, então $h^{-1}(A)$ está contido em $\operatorname{DOM}(T)$;

(a2) Seja $\bar{p} \in \overline{\gamma_{p}}$ um ponto recorrente não-trivial de $X$ e $(\bar{p},[\bar{p}, \bar{q}))$ um par satisfazendo as mesmas condições que $(p,[p, q))$ acima. Então a propriedade da IET $E_{(\bar{p},[\bar{p}, \bar{q}))}$ pertencer a $\mathcal{B}_{k}$ não depende de $(\bar{p},[\bar{p}, \bar{q}))$, mas sim de $X$.

Sob as condições do teorema acima, e se $E \in \mathcal{B}_{k}$, qualquer ponto recorrente não-trivial de $\overline{\gamma_{p}}$ é denominado ponto de tipo $\mathcal{B}_{k}$. O teorema $B$ é uma combinação do teorema de estrutura, do fato de $\mathcal{B}$ ter medida total no espaço das IET's (lema 2.3.1), e do teorema abaixo:

Teorema 3.2.3. Sejam $X \in \mathcal{X}^{r}(M), 1 \leq \infty$, tendo $K \geq 0$ singularidades. Seja $p \in M$ um ponto recorente não-trivial de tipo $\mathcal{B}_{k}$. Então existe um $Y \in X^{r}(M)$, arbitrariamente próximo de $X$, possuindo uma trajetória fechada passando por $p$.

\section{Demonstração}

Este teorema é enunciado da seguinte forma: seja $p \in M$ ponto recorrente não-trivial de tipo $\mathcal{B}_{k}$ de $X \in X^{r}(M), 1 \leq r \leq \infty$. Suponha que $X$ tenha $K \geq 0$ singularidades. Então existe um $Y \in X^{r}(M)$ arbitrariamente próximo de $X$, tendo uma trajetória fechada passando por $p$.

Pelo teorema 3.0.2, é suficiente mostrar que $X$ é $\Sigma$-fechado em $p$. Sejam $\Sigma=[p, q), T:[p, q) \rightarrow$ $[p, q), E:[0,1) \rightarrow[0,1), h:[p, q) \rightarrow[0,1)$, como no teorema de estrutura. No lema 2.3.1, o fato de que $\lim _{n} a_{n}=0$, nos permite tomar $n$ grande o suficiente, de forma que $h^{-1}\left(\left[0, a_{n}\right)\right)$ esteja arbitrariamente próximo de $p$. Defina $b=a_{n}$. Então:

Como $E \in \mathcal{B}_{k}$, dada uma vizinhança $V$ de $p$, existem $b \in(0,1)$ e uma IET $E_{V}:[0, b) \rightarrow[0, b)$ tais que (d) $E_{V}$ tem ao menos $\chi+K+3$ arestas virtuais ortogonais contidas em $[0, b)$, e além disto, o intervalo $h^{-1}([0, b))$ está contido em $V$. Seja $T_{V}: \Sigma_{V} \rightarrow \Sigma_{V}$ a transformação de primeiro retorno induzida por $T$. Como $X$ tem $K$ singularidades, (d) e o teorema de estrutura implicam que $E_{V}$ possui uma aresta virtual ortogonal $\left[a, E_{V}(a)\right] \subset[0, b)$ tal que para algum $a \in \operatorname{Dom}\left(\left.T\right|_{\Sigma_{V}}\right),\left[\bar{a}, T_{V}(\bar{a})\right]=h^{-1}([a, E(a)]) \subset \operatorname{Dom}\left(\left.T\right|_{\Sigma_{V}}\right)$. Portanto, existe uma $\Sigma$-caixa de fluxo limitada por $\left[\bar{a}, T_{V}^{2} \bar{a}\right] \cup\left[\bar{a}, T_{V}^{2}(\bar{a})\right]_{X}$. Como $V$ foi escolhido de forma arbitrária, isto mostra que $X$ é $\Sigma$-fechado em $p$. 


\section{Apêndice A}

\section{Noções básicas da Teoria dos Sistemas Dinâmicos}

Um sistema dinâmico é uma construção que sugere uma ordenação temporal sobre o espaço, onde o tempo é representado por um elemento de um grupo aditivo $G=\mathbb{Z}$, ou $G=\mathbb{R}$. Como veremos, os fluxos definidos por campos vetoriais e as iterados de uma IET definem sistemas dinâmicos.

Definição A.0.1. Sejam $X$ um espaço topológico e $\phi: G \times X \rightarrow X$ uma transformação tal que

1. $\phi(\phi(x, t), s)=\phi(x, t+s), \forall x \in X e \forall s, t \in G$.

2. $\phi(x, 0)=x, \forall x \in X$.

Dizemos que o par ordenado $(X, \phi)$ é um sistema dinâmico sobre $X$.

Observação A.0.1. A definição acima pode ser estendida para o caso em que $G$ é apenas um grupo topológico, como por exemplo, um grupo de Lie. Desta forma, substituimos o 0 na definição acima pelo elemento neutro $e \in G$. Aqui consideraremos os seguintes casos:

- $G=\mathbb{R}$ : diremos que $\phi$ é um fluxo sobre $X$ e que $(X, \phi)$ é um sistema dinâmico contínuo.

- $G=\mathbb{Z}$ : diremos que $(X, \phi)$ é um sistema dinâmico discreto.

Os sistemas discretos aqui citados serão aqueles definidos por iterações de uma transformação invertivel $T: X \rightarrow X$ da seguinte forma: $\phi(x, k)=T^{k}(x), \forall x \in X$ e $\forall k \in \mathbb{Z}$. É fácil ver que $\phi$ definida desta forma satisfaz as propriedades necessárias para que $(X, \phi)$ seja um sistema dinâmico discreto, que será denotado por $(X, T)$ para simplificar a notação.

Exemplo Sejam $M$ uma variedade suave e compacta de dimensão $n \in \mathbb{N}$. Seja $X: M \rightarrow T M$ um campo vetorial de classe $C^{1}$. Então pelo teorema de existência e unicidade, para todo $x \in M$ existe uma solução global $\varphi_{x}: \mathbb{R} \rightarrow M$ da equação $\dot{x}=X(x)$. A função $\varphi: \mathbb{R} \times M \rightarrow M$ dada 
por $\varphi(t, x)=\varphi_{x}(t)$ é um fluxo, e portanto $(M, \varphi)$ é um sistema dinâmico. Para $t \in \mathbb{R}$ fixo, $\varphi$ define um sistema dinâmico discreto $(X, f)$ da seguinte forma: $f(k, x)=\varphi(t k, x)$, para $k$ inteiro e $x$ em $X$.

Definição A.0.2. O conjunto $\{(x, t)\}_{t \in} \subset X$ é chamado de órbita de $x$, e aqui será denotado por $\mathcal{O}(x)$. Se existe $T \in G$ tal que $(x, t+T)=x$, dizemos que x e sua órbita são T-periódicos. O menor $T$ tal que $x$ é T-periódico é chamado de período de $x$. Se o período de $x$ for 1 , dizemos que $x$ é ponto fixo. Nesta caso $\mathcal{O}(x)=\{x\}$.

Definição A.0.3. Para todo $x \in X$, definimos os seguintes subconjuntos (possivelmente vazios) de $X: \alpha(x)=\left\{x \in Z\right.$ tal que $\exists\left\{t_{N}\right\}_{n \in \mathbb{N}}$ satisfazendo $\lim _{n} t_{n}=-\infty$ e $\left.\lim _{n}\left(t_{n}, x\right)=x\right\}$

Definição A.0.4. Dizemos que um ponto $p \in X$ é não-errante caso para toda vizinhança $V$ de $p, \exists T=T(V) \in G, T>0$ tal que $\{(\{x\} \times V)\} \cap V \neq \emptyset$.

Definição A.0.5. Dizemos que um ponto $p \in M$ é recorrente é errante caso $x \in \alpha(x)$ ou $x \in \omega(x)$.

Observação A.0.2. Se $X$ for um espaço topológico primeiro contável, um ponto $p \in X$ é nãoerrante caso $\exists p_{n_{n \in \mathbb{N}}}$ sequência de pontos de $X$ e $t_{n}$ sequência de números reais tal que $t_{n} \rightarrow \infty$ quando $n \rightarrow \infty$, tais que $\left(p, t_{n}\right) \rightarrow p$, quando $n \rightarrow \infty$. Segue que se pudermos tomar $p_{n}=p$ para todo $n \in \mathbb{N}$, então p é ponto recorrente.

Proposição A.0.1. Sejam $M$ uma variedade compacta e suave, $X \in \chi^{r}(M), r \geq 1$ um campo vetorial e o fluxo gerado por este campo. Se $\Omega(X)$ é o conjunto dos pontos não-errantes de, então $\Omega(X)$ é um compacto não-vazio e contém o conjunto dos ponto recorrentes, que por sua vez contém $\operatorname{Per}(X)$, o conjunto dos pontos periódicos de.

Lema A.0.1. Se $p \in M$ é ponto recorrente não-trivial, então qualquer ponto em $\gamma_{p}$ também é.

Demonstração Basta mostrar que $p$ é recorrente, já que ele não é ponto periódico por estar na órbita de um ponto não-periódico de um fluxo. Suponha que $p$ é recorrente. Então $p \in \omega(p)$, sem perda de generalidade, pois se $p \in \alpha(X)$, então $p \in \omega(-X)$.

Por sua vez, como $x \in \gamma_{p}, x$ é da forma $\varphi(t, p)$, para algum $t$ real. $p \in \omega(p)$ implica que existe uma seqüência $\left\{t_{n}\right\}$ tal que $\lim _{n} t_{n}=+\infty$ e que satisfaz $\lim _{n} \varphi\left(t_{n}, p\right)=p$. Pela continuidade de $\varphi_{t}, x=\varphi(t, p)=\varphi\left(t, \lim _{n} \varphi\left(t_{n}, p\right)\right)=\lim _{n} \varphi\left(t, \varphi\left(t_{n}, p\right)\right)=\lim _{n} \varphi\left(t_{n}, \varphi(t, p)\right)=\lim _{n} \varphi\left(t_{n}, x\right)$. Logo $x \in \omega(x)$, e portanto $p$ é ponto recorrente não-trivial.

Definição A.0.6. Uma transformação $T: X \rightarrow X$ invertível é dita minimal se para todo $x \in X$, sua órbita $\mathcal{O}(x)$ seja densa em $X$. 


\section{Apêndice B}

\section{Noções básicas da Teoria Ergódica}

Nesta apêndice reunimos alguns fatos básicos da teoria érgodica que são utilizados no texto. Utilizaremos as letras $\left(X, \mathcal{U}, d \mu_{1}\right),\left(Y, \mathcal{V}, d \mu_{2}\right)$ para denotar $\sigma$-álgebras.

Definição B.0.7. Dizemos que uma transformação $f: X \rightarrow Y$ é mensurável caso $f^{-1}(V)$ pertença a $\mathcal{U}$ para todo $V \in \mathcal{V}$.

Definição B.0.8. Dizemos que uma transformação $f: X \rightarrow Y$ preserva medida caso $\mu_{1}\left(T^{-1}(V)\right)=$ $\mu_{2}(V)$ para todo $U \in \mathcal{V}$. Se $X=Y$, dizemos que uma medida $\mu$ é $f$-invariante caso $\mu_{1}\left(T^{-1}(U)\right)=$ $\mu_{1}(U)$ para todo $U \in \mathcal{U}$.

Definição B.0.9. Dizemos que uma transformação $f: X \rightarrow X$ que preserva medida é ergódica caso $T^{-1}(U)=U$ se e somente se $U=X$ ou $U=\varnothing$.

Lema B.0.2. Se existe exatamente uma medida f-invariante, enão esta medida é ergódica, e é chamada de unicamente ergódica.

Teorema B.0.4 (Teorema Ergódico de Birkhoff). Se T $:(M, \mu) \rightarrow(M, \mu)$ é uma transformação ergódica que preserva medida num espaço de probabilidade $(M, \mu)$. Se $A \in M$ for um conjunto mensurável, então para todo $x \in M$ a menos de um conjunto de medida zero,

$$
\mu(A)=\lim _{N \rightarrow \infty} \frac{1}{N} \sum_{j=0}^{N-1}\left\{j \in\{0,1, \ldots, N-1\} \mid T^{j}(x) \in A\right\}
$$

Se $X$ for um espaço métrico e $T$ for unicamente ergódica, então a convergência acima vale uniformemente e em toda a parte. 


\section{Referências Bibliográficas}

[Gu1] Gutierrez C. 1978 Smooth non-orientable non-trivial recurrences on two-manifolds, J. Diff. Eqns., 3, 388-95.

[Gu2] Gutierrez C. 1987 A counter-example to a $C^{2}$-closing lemma, Erg. Theor. Dyn. Sys., 7, 509-30.

[Gu3] Gutierrez C. 1986 On the $C^{r}$-closing lemma for flows on the torus $T^{2}$, Erg. Theor. Dyn. Sys., 6, 45-56 .

[Gu4] Gutierrez C. 1986, Smoothing continuous flows on two-manifolds and recurrences, Erg. Theor. Dyn. Sys., 6, 17-44.

[Gu5] Gutierrez C. 1978, Structural stability for flows on the torus with a cross-cap, Trans. Amer. Math. Soc., 241, 311-20.

[Gu6] Gutierrez C. 2000, On $C^{r}$-closing for flows on two-manifolds, Nonlinearity, 241, 1883-88.

[Man] Mañé R. 1982, An ergodic closing lemma, Ann. of Math, 116, 503-41.

[Mar] Markley N. 1969 The Poincaré-Bendixon theorem for the Klein bottle, Trans. Am. Math. Soc., 135, 159-65.

[Mas] Masur H. 1982, interval exchange transformations and measured foliations, Ann. Math., 115, 169-200.

[Pe1] Peixoto M. 1987, Accptance speech for the TWAS award in mathematics, Proc. of the Second General Conference Organized by the Third World Academy of Sciences, 1, 606 .

[Pe2] Peixoto M. 1962, Structural stability on two-dimensional manifolds, Topology, 1, 101-20.

[Pg1] Pugh C. 1967 The Closing Lemma, Am. J. Math., 89, 956-1009.

[Pg2] Pugh C. 1967 An improved closing lemma and a general density theorem, Am. J. Math., 89, 1010-21. 
[Sl] Slater N. B. 1967, Gaps and steps of the sequence nOmod1, Proc.Camb.Phil.Soc., 63, 1115123.

[S-T] Schwatz A. J. Thomas E. S. 1970, The depth of the center 2-manifolds, Global Anal. Pure Math., 14, Amer. Math. Soc.: Providence, R.I., 253-64.

[Vee] Veech W. 1982, Gauss measure for transformations on the space of interval exchange maps, Ann. Math., 115, 201-42. 